\title{
Selective determination of doxorubicin and doxorubicinol in rat plasma by HPLC with photosensitization reaction followed by chemiluminescence detection
}

\author{
Sameh Ahmed, Naoya Kishikawa, Kaname Ohyama, Mitsuhiro Wada, Kenichiro \\ Nakashima and Naotaka Kuroda* \\ Graduate School of Biomedical Sciences, Course of Pharmaceutical Sciences, \\ Nagasaki University, 1-14 Bunkyo-machi, Nagasaki 852-8521, Japan
}

*Corresponding author;

E-mail address: n-kuro@ nagasaki-u.ac.jp

Tel: $+81-95-8192894$

Fax: +81-95-8192444 


\begin{abstract}
A highly sensitive and selective high-performance liquid chromatography (HPLC) method was developed for the determination of doxorubicin (DXR) and its metabolite doxorubicinol (DXR-ol) in rat plasma. The method was based on photosensitization reaction followed by peroxyoxalate chemiluminescence detection (PO-CL). DXR and DXR-ol that were fluorescent quinones, served as a photosensitizer in presence of a hydrogen atom donor such as ethanol under aerobic conditions to produce hydrogen peroxide. Then the generated hydrogen peroxide and DXR or DXR-ol were monitored through PO-CL reaction by mixing with aryloxalate as a single post-column reagent that enabled highly selective and sensitive determination of DXR and DXR-ol. The separation of DXR and DXR-ol by HPLC was accomplished isocratically on an ODS column within 15 min. The method involves a simple one step protein precipitation by methanol and a sample size of $50-\mu \mathrm{L}$ was sufficient. Besides, it can detect accurately the low plasma concentrations. The detection limits (signal-to-noise ratio $=3$ ) were 4.5 and $3.8 \mathrm{fmol}$ for DXR and DXR-ol, respectively. The percentage recovery was found to be 90.7-102.4\% and the inter- and intra- assay RSD values in rat plasma were 2.5-8.9 \%. The method has been successfully used to study pharmacokinetic profiles of DXR and DXR-ol in rats after a single dose of DXR.
\end{abstract}

Keywords: Doxorubicin, Doxorubicinol, Pharmacokinetics, Peroxyoxalate chemiluminescence detection, Photosensitization. 


\section{Introduction}

Doxorubicin (DXR) is a cytotoxic anthracycline antibiotic that is originally produced by Streptomyces peucetius var. caesius and has been used in the treatment of a wide range of malignant tumours, including leukemia and breast cancer. However, the clinical use of doxorubicin is limited by a cumulative dose-dependent irreversible chronic cardiomyopathy, which can subsequently lead to congestive heart failure, with an ultimate mortality rate of 20-40\%. Moreover, DXR-induced cardiotoxicity may be reduced or prevented by an administration schedule that produces low peak plasma drug concentrations [1,2].

DXR is rapidly metabolized in liver by the cytoplasmic NADPH-dependent aldo-keto reductase to the secondary alcohol metabolite, doxorubicinol (DXR-ol), and by the NADPH dependent cytochrome P450 reductase to a broad panel of hydroxy- or deoxy-aglycones. Of these metabolites, DXR-ol has been demonstrated to be cardiotoxic. However, the role of doxorubicin aglycones in the cardiotoxicity remains unclear (Fig.1) [3].

To date, a number of publications have been reported of the determination of DXR and its metabolite DXR-ol including capillary electrophoresis (CE) with UV detection [4] or laser-induced fluorescence (LIF) detection [5-7], radioimmunoassay [8], high performance liquid chromatography (HPLC) with ultraviolet (UV) detection [9-10], fluorescence detection [11-21], chemiluminescence detection [22], electrochemical detection [23] and mass spectrometric detection [25-29]. However, many of these methods lack the enough sensitivity and selectivity which enable the determination of DXR and DXR-ol at small plasma concentration while the high cost and the need for technical experience limits the use of the others. In addition, some of these techniques are laborious and necessitate long analytical run times to achieve sufficient peak resolution, owing to structural similarities of DXR and DXR-ol. Besides, sample pre-treatment procedures in these methods vary greatly. Some of them include time-consuming solid-phase extraction processes [23-26], or liquid-liquid extraction procedures $[4,12,17,20,28]$. The instability of DXR and DXR-ol in plasma also limits the utility of otherwise promising extraction strategies. From a practical point of view, a simple and rapid sample pre-treatment procedure is desirable in a single protein precipitation step. 
Peroxyoxalate chemiluminescence (PO-CL) is based on the reaction between hydrogen peroxide and aryloxalate, which produces strong luminescence in the presence of fluorophore through the CIEEL (chemically initiated electron exchange luminescence) mechanism [30]. The PO-CL is easily combined with HPLC and applied to highly sensitive determination of fluorescent compounds and hydrogen peroxide. Previously, we had reported the determination of quinones by HPLC-PO-CL detection, without addition of hydrogen peroxide and fluorophore, combined with on-line photodegradation reaction [31] and this method was further adopted for the determination of vitamin $\mathrm{K}$ homologues [32]. This method was based on the production of hydrogen peroxide and conversion of non-fluorescent quinones to a fluorescent 3,6-dihydroxyphthalic acid through photodegradation reaction by UV irradiation, and then detected via PO-CL reaction by mixing with aryloxalate. Furthermore, it was reported that quinones served as sensitizer for photooxidation of hydrogen atom donor (HAD) under aerobic conditions to generate hydrogen peroxide and quinones were regenerated [33-34]. Moreover, DXR and DXR-ol are highly fluorescent quinones and can be used as fluorophore for PO-CL reaction after photosensitization reaction because they were regenerated along with the production of hydrogen peroxide.

Therefore, we aimed in this research to develop an HPLC method for their determination based on their unique photochemistry with UV irradiation $(254 \mathrm{~nm}, 15 \mathrm{~W})$ of DXR or DXR-ol in presence of ethanol as an HAD under aerobic conditions to generate hydrogen peroxide followed by chemiluminescence reaction by mixing with aryloxalate. The use of aryloxalate as a single post-column reagent provides advantages on the stability of CL reagent and subsequently reproducibility of the method in addition to the expected decrease of background noise. As a general case in PO-CL reaction, a mixture of aryloxalate together with hydrogen peroxide or a fluorophore is used as post-column CL reagent, which suffers from stability problems accompanied with high background noise. Interestingly, only fluorescent compounds with quinone moiety can give a response in our method that imparts the high selectivity that enabled us to determine small sample concentrations without any interference from plasma components. 


\section{Experimental}

\subsection{Chemicals and reagents}

Doxorubicin hydrochloride was obtained from Wako Pure Chemical Industries (Osaka, Japan). Doxorubicinol hydrochloride was from Toronto Research Chemicals, Inc. (Toronto, Ontario, Canada). Stock solutions of DXR and DXR-ol $(100 \mu \mathrm{M})$ were prepared in methanol and stored in the dark at $-30{ }^{\circ} \mathrm{C}$. These solutions were diluted appropriately with methanol to prepare the working solutions. Bis[2-(3,6,9-trioxadecyloxycarbonyl)-4-nitrophenyl]oxalate $\quad$ (TDPO), Bis[2-(3,6dioxaoctyloxycarbonyl)-4-nitrophenyl]oxalate (DOPO) and sodium dodecyl sulphate (SDS) were from Wako Pure Chemical Industries. Bis(2,4,6-trichlorophenyl)oxalate (TCPO), bis[(2,4,5-trichloro-6-pentoxycarbonyl) phenyl] $]$ oxalate (CPPO), bis(2,4dinitrophenyl)oxalate (DNPO), bis(2,6-difluorophenyl)oxalate (DFPO) and bis(pentafluorophenyl)oxalate (PFPO) were from Tokyo Chemical Industry (Tokyo). Acetonitrile (LC/MS grade) were from Kanto Chemical (Tokyo). Imidazole was from Tokyo Chemical Industry; imidazole was recrystallized from acetonitrile before use. Distilled water was obtained using Simpli Lab-UV (Millipore, Bedford, MA, USA) water device. Other chemicals were of extra pure grade. All centrifuge tubes and HPLC vials were made of polypropylene to minimize adsorption effects of DXR and DXR-ol.

\subsection{HPLC-PO-CL system}

The HPLC system (Fig. 2) consisted of two LC-10AS liquid chromatographic pumps (Shimadzu, Kyoto, Japan), a Rheodyne 7125 injector (Cotati, CA, USA) with a $5-\mu \mathrm{L}$ sample loop, a Cosmosil 5C18-AR-II (150 x 2 mm, i.d., Nacalai Tesque Inc., Kyoto) column, a low-pressure mercury lamp (15 W, 254 nm, Sigemi Standard, Tokyo), a CLD-10A chemiluminescence detector (Shimadzu) and SIC chromatorecorder (Tokyo, Japan). PTFE tubing ( $2.0 \mathrm{~m}$ x $0.25 \mathrm{~mm}$ i.d., GL Sciences, Tokyo) coiled around a low-pressure mercury lamp was used as on-line photoreactor. Temperature of the photoreactor was maintained at $50{ }^{\circ} \mathrm{C}$ by an oven (Jasco, Tokyo). A mixture of imidazole-trifluoroacetic acid (TFA) buffer (50 mM, pH 6.8), acetonitrile and ethanol (55:35:10, v/v/v) containing $20 \mathrm{mM}$ SDS was used as a mobile phase and $0.5 \mathrm{mM}$ TDPO in acetonitrile was used as the post column CL 
reagent. The mobile phase and CL reagent were filtered through a $0.45-\mu \mathrm{m}$ filter prior to use. The flow-rates of the mobile phase and the CL reagent were set at 0.3 and $0.7 \mathrm{~mL} \mathrm{~min}^{-1}$, respectively.

\subsection{Experimental animals}

Wistar male rats (285-317 g, Tagawa experimental animals, Nagasaki, Japan) were used for experiments. Animals were housed separately in a metabolic cage and kept on a 12-h light/dark cycle for two days before being used in experiment. Food and water were available ad libitum. Rats were anesthetized with ethyl carbamate i.p. (1.5 g/kg). DXR was administered (i.a.) via arteria femoralis to a group of three male Wistar rats at a dose of 5 $\mathrm{mg} / \mathrm{kg}$. Blood samples $(150-\mu \mathrm{L})$ were collected through indwelling arterial catheters, transferred to EDTA tubes and centrifuged for $10 \mathrm{~min}$ at $4{ }^{\circ} \mathrm{C}, 5000 \mathrm{x} g$ then the plasma was separated and stored at $-30{ }^{\circ} \mathrm{C}$ in dark until analysis. Sampling of blood was performed prior administration of DXR and then at 10, 20, 30, 45, 60, 120, 180, 240, 300, 360 and $420 \mathrm{~min}$ after administration of DXR. All animal procedures and care in this experiment were approved by the Nagasaki University Animal Care and Use Committee (approval no. 0807010676). Pharmacokinetic parameters were determined by moment analysis using a single-dose i.a. bolus drug adminstration model. The studied parameters are area under the curve (AUC), terminal phase half life $\left(\mathrm{T}_{1 / 2}\right)$, clearance $(\mathrm{CL})$, maximum concentration $\left(\mathrm{C}_{\max }\right)$ and volume of distribution at steady state $\left(\mathrm{V}_{\mathrm{dss}}\right)$.

\subsection{Sample processing}

Sample preparation involved a single protein precipitation step. A $50-\mu \mathrm{L}$ aliquot of plasma were pipetted into polypropylene Eppendorf micro test tube (Hamburg, Germany), followed by $150-\mu \mathrm{L}$ methanol for protein precipitation. The tube was vigorously mixed for $30 \mathrm{~s}$ and centrifuged for $10 \mathrm{~min}$ at $10000 \mathrm{xg}$. A 5 - $\mu \mathrm{L}$ supernatant was injected directly into the HPLC system.

\subsection{Method validation}


The validation was done according to US FDA guidance for industry on bioanalytical method validation [35].

Three days of calibration curves with five replicates of quality controls (QC) over concentration range of 2-1000 $\mathrm{nM}$ were performed to assess linearity of the proposed method.

To evaluate intra-day and inter-day precision and accuracy, three sets of QC samples at three different concentration levels low-QC (50 nM), middle-QC (250 nM) and high-QC $(1000 \mathrm{nM})$ with five replicates at three successive days. The samples were extracted by single protein precipitation step with methanol then $5 \mu \mathrm{L}$ of supernatant was injected directly to the HPLC system.

Pre-spiked plasma samples were used for the recovery study. The recovery was determined in triplicate at three concentration levels $50 \mathrm{nM}, 250 \mathrm{nM}$ and $1000 \mathrm{nM}$ by extraction of drug-free rat plasma fortified with standard DXR and DXR-ol. The fortified extracts were injected into HPLC system. The obtained results were compared with true concentration of the pure standard.

Finally, the stability of DXR and DXR-ol in rat plasma under various laboratory conditions was investigated in triplicate in both low-QC (50 nM) and high-QC (1000 nM). The quality control samples were freshly prepared and subjected to three-times freeze cycles, storing at room temperature for 1,5 and $24 \mathrm{~h}$, refrigeration overnight at $4{ }^{\circ} \mathrm{C}$ and storing in freezer at $-30{ }^{\circ} \mathrm{C}$ for three weeks. The samples were extracted and analyzed by the proposed method.

\section{Results and discussion}

\subsection{Photosensitization reaction of doxorubicin and its metabolite doxorubicinol}

Photochemical reaction between anthraquinone or hydroxyanthraquinones and hydrogen atom-donating substrates has been widely studied [33,36]. Quinone sensitizers serve as catalysts for the reduction of oxygen and the oxidation of HAD substrates. Similarly, DXR and DXR-ol act as sensitizers for photooxidation of HAD such as ethanol. DXR is excited to its singlet-excited state (S1) by UV irradiation and most of the excited DXR is converted by intersystem crossing (ISC) to the triplet state and subsequent by internal conversion to its lowest triplet state (T1). Next, the excited DXR in the triplet state (T1) abstract hydrogen 
from ethanol to produce two radical products, the semiquinone form of DXR and $\alpha$-hydroxyethyl radical. Under aerobic conditions these two radical intermediates react with molecular oxygen to produce hydrogen peroxide and DXR is regenerated (Scheme 1).

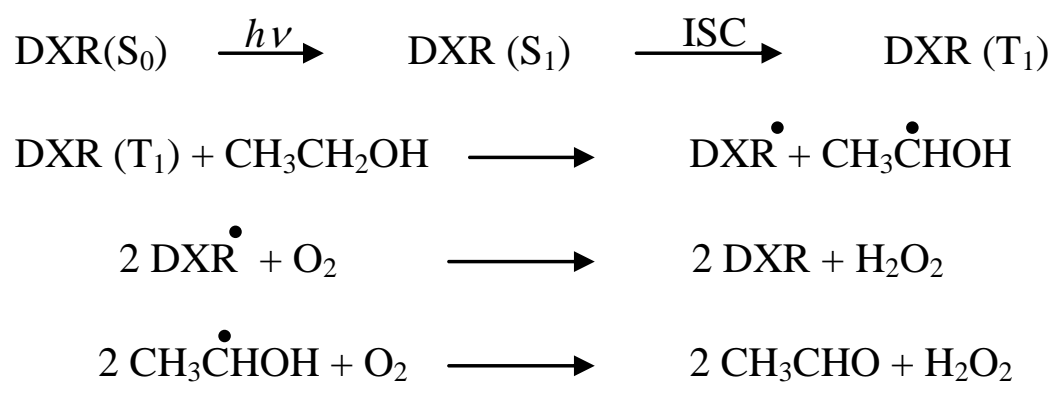

\subsection{Optimization of HPLC-PO-CL method}

Since it was necessary to develop a highly sensitive and selective method for determination of DXR and DXR-ol at therapeutic level of the drugs in plasma, the conditions for separation and determination of DXR and DXR-ol with the proposed method were optimized in order to obtain the best resolution and sensitivity.

\subsubsection{Optimization of separation and photochemical reaction conditions}

Isocratic elution mode was employed for resolution and quantitation of DXR and DXR-ol. A mixture of imidazole buffer, acetonitrile and ethanol (55:35:10, v/v/v) containing SDS was used as a mobile phase. SDS was selected as ion-pair reagent not only for its effect on separation and peak shape of DXR and DXR-ol [10] but also for its enhancement and stabilization effect on PO-CL reaction [37]. Furthermore, SDS has a hydrogen atom-donating ability [38]. The influence of SDS concentration on separation and peak shape of DXR and DXR-ol peaks was tested. It was found that above its critical micellar concentration, reproducible and stable signals were obtained probably due to the protection of aryloxalate (TDPO) molecules in the micelles, thereby avoiding decomposition processes. In addition, the separation and peak symmetry were largely improved. Given this effect, a concentration of $20 \mathrm{mM}$ was selected as optimum.

The presence of HAD in mobile phase such as methanol, ethanol and isopropanol was also studied (Fig. 3). Nevertheless, the presence of SDS in mobile phase, presence of hydrogen atom donor enhance greatly CL intensity probably due to its effect on the amount 
of generated hydrogen peroxide. Ethanol was selected because it is effective as an enhancer of CL intensity and as eluent for DXR and DXR-ol. Moreover, the concentration of ethanol was studied and the best separation and CL intensity was obtained at ethanol concentration of $10 \%(\mathrm{v} / \mathrm{v})$.

Furthermore, effect of photoreactor conditions such as UV irradiation lamps, the length of the reaction coil wrapped around UV lamp and photoreactor temperature were investigated. Three types of UV lamps were evaluated as UV sources; Toshiba GL-10 (10 W, $254 \mathrm{~nm}$ ), Shigemi standard AL-15 H (15 W, $254 \mathrm{~nm})$ and National FL-10 BL-B (10 W, 350 $\mathrm{nm}$ ). We found that $15 \mathrm{~W}$ powered lamp, which emits at $254 \mathrm{~nm}$ wavelengths, gave higher $\mathrm{CL}$ intensity and signal-to-noise $(\mathrm{S} / \mathrm{N})$ ratio than the other two lamps. Therefore, it was chosen for subsequent work.

The effect of coil length ranging from 0.5 to $4.0 \mathrm{~m}$ with $0.25 \mathrm{~mm}$ internal diameter corresponding to 5 - 40 seconds of UV irradiation on CL intensity of DXR and DXR-ol was examined. It was found that CL intensity and $\mathrm{S} / \mathrm{N}$ increased as the length of the reaction coil increased due to the increase in exposure time then decreased probably due to the decomposition of hydrogen peroxide by long UV irradiation time. From this experiment, 2.0 $\mathrm{m}$ of coil length, corresponding to 20 seconds of UV irradiation, was selected as optimal.

In addition, the influence of photoreactor temperature between $20^{\circ} \mathrm{C}$ and $60^{\circ} \mathrm{C}$ on $\mathrm{CL}$ of DXR and DXR-ol was investigated. It was found that both CL intensity and S/N ratio increased by the increase in temperature till $50^{\circ} \mathrm{C}$ then decreased. This is probably due to the increase in the amount of generated hydrogen peroxide by the rise in temperature then the more rise in temperature lead to increase decomposition of hydrogen peroxide. Therefore, $50{ }^{\circ} \mathrm{C}$ was selected.

\subsubsection{Optimization of CL conditions}

Imidazole has been reported as the most efficient catalyst for the PO-CL reaction, acting not only through a simple base catalysis but also nucleophilic catalysis pathway [30]. In this study, we have investigated the effect of different acids used in combination with imidazole. Several acids were tried such as $\mathrm{HNO}_{3}, \mathrm{HClO}_{4}, \mathrm{H}_{3} \mathrm{PO}_{4}, \mathrm{HCl}, \mathrm{H}_{2} \mathrm{SO}_{4}$ and TFA. However, the highest $\mathrm{CL}$ intensity and $\mathrm{S} / \mathrm{N}$ ratio was obtained when using a combination of imidazole-TFA. This may be attributed to the effect of TFA on PO-CL reaction kinetics and stability of CL reagent [39]. Next, the influence of imidazole-TFA buffer solution on CL 
intensity of DXR and DXR-ol was examined. It was found that the best CL intensity and $\mathrm{S} / \mathrm{N}$ ratio were obtained at $50 \mathrm{mM}$ imidazole-TFA buffer solution. Subsequently, the influence of $\mathrm{pH}$ of the imidazole-TFA buffer solution on CL was studied. CL intensity increased with the increase in $\mathrm{pH}$ value till 7.5 then decreased, which could be attributed to instability of DXR and DXR-ol at higher $\mathrm{pH}$. Although, S/N ratio was decreased after $\mathrm{pH}$ 6.8 and therefore $\mathrm{pH}$ value of 6.8 was selected for subsequent work (Fig.4).

In order to increase the quantum yield and light intensity produced for CL reaction of DXR and DXR-ol, we aimed to study the effect of different aryloxalates on CLintensity and $\mathrm{S} / \mathrm{N}$ ratio of DXR and DXR-ol. Because it was reported that aryloxalates with strongly electron-withdrawing substituents provided the highest quantum yields [38], therefore we have tested; TCPO, TDPO, DNPO, DOPO, DFPO, PFPO and CPPO as the most efficient PO-CL reagents. We found that TDPO gave the best CL intensity and S/N ratio for both DXR and DXR-ol. Moreover, TDPO provides improved solubility (1010 mM) and chemical stability in acetonitrile relative to other aryloxalates that further provides superior reproducibility [40].

Furthermore, the effect of TDPO concentration on CL on DXR and DXR-ol was studied. It was found that CL intensity increases by increasing the concentration of TDPO whereas $\mathrm{S} / \mathrm{N}$ ratio increases till $0.5 \mathrm{mM}$ then decreased. The reason for this is the increase in background noise. Therefore, the concentration of $0.5 \mathrm{mM}$ TDPO was selected for subsequent work. Subsequently, effect of TDPO flow rate on CL of DXR and DXR-ol was investigated. It was found that CL intensity increased by the increase in flow rate and reached maximum at $0.70 \mathrm{~mL} \mathrm{~min}^{-1}$ then $\mathrm{CL}$ intensity decreased due to the decrease in reaction time between CL reagent, hydrogen peroxide and the analyte. From this experiment, flow rate of $0.70 \mathrm{~mL} \mathrm{~min}^{-1}$ was selected as optimal.

\subsection{Validation of the proposed method}

Under the optimum experimental conditions, linear relationship was observed by plotting drug concentration ( $\mathrm{nM}$ ) against relative chemiluminescence intensity (RCI). Calibration curve, retention time, calibration range, correlation coefficient and detection limit were recorded for DXR and DXR-ol (Table 1). The correlation coefficients of 0.998 or more were obtained in the concentration ranges of $2-1000 \mathrm{nM}$ for DXR and DXR-ol which indicate the good linearity of calibration graphs. Also, the detection limits obtained with the 
proposed method $(\mathrm{S} / \mathrm{N}=3)$ were 4.5 and 3.8 fmol for DXR and DXR-ol, respectively. A typical chromatogram of a standard mixture of DXR and DXR-ol using the proposed method is shown in Fig. 5 (A). DXR and DXR-ol were fully separated and eluted within 15 minutes. Accuracy and precision of the proposed method were assessed at three different concentration levels; low $(50 \mathrm{nM})$, middle $(250 \mathrm{nM})$ and high $(1000 \mathrm{nM})$ concentrations at three different days (Table 2). It was found that the accuracy of the determination was 92.3101.5\% and 90.4-102.5\% for DXR and DXR-ol, respectively. The relative standard deviations (RSD) intra-day $(n=5)$ and inter-day $(n=3)$ were $3.5-8.9 \%$ and $2.5-7.6 \%$ for DXR and DXR-ol, respectively so good reproducibility was obtained. Moreover, extraction recovery was established with three different concentration levels; low (50 nM), middle $(250 \mathrm{nM})$ and high $(1000 \mathrm{nM})$. The percentage recovery was found to be $91.2-96.7 \%$ and 90.7-102.4\% for DXR and DXR-ol, respectively. These results indicate the high efficiency of the extraction procedure in addition to its rapidity and simplicity. Besides, there is no matrix effect as the extracted samples can be determined without any interference from plasma matrix components that emphasize the bio-specificity of the proposed method.

The stability experiments of DXR and DXR-ol were carried out under various laboratory conditions such as three-times freeze-thaw stability, after storing at room temperature for 1 , 5 and $24 \mathrm{~h}$, refrigeration overnight at $4{ }^{\circ} \mathrm{C}$ and freezer stability at $-30{ }^{\circ} \mathrm{C}$ for three weeks (Table 3). The variations of DXR and DXR-ol concentration in rat plasma for the freeze-thaw operations, room temperature stability for $1 \mathrm{hr}$ and $5 \mathrm{hr}$, refrigerator stability and freezer stability were within $13 \%$. These results met with the acceptance criteria (+ $15 \%$ ) according to US FDA guidance [35]. However, $24 \mathrm{hr}$ room temperature storage led to about $20 \%$ loss. Therefore, DXR and DXR-ol samples should be stored in cold as soon as possible, which is consistent with previous reports [11,24].

\subsection{Application to pharmacokinetic studies of doxorubicin and its metabolite in rat}

In order to test the applicability of the method to measure DXR and DXR-ol in the course of pharmacokinetic studies, the proposed HPLC-PO-CL method was used for simultaneous determination of DXR and DXR-ol in rat plasma after a single dose administration of DXR. The smaller amount of plasma required for the determination enable many sampling points. DXR and DXR-ol were detected in rat plasma up to 420 min after administration of DXR. A typical chromatogram of rat plasma collected after $60 \mathrm{~min}$ of single dose administration of 
DXR is shown in Fig. 5 (B) that fully confirms the high selectivity of the proposed method. Concentration-time profiles of DXR and DXR-ol in rat plasma after a single dose administration of $5 \mathrm{mg} / \mathrm{kg}$ DXR are presented in Fig. 6. The pharmacokinetic parameters (mean $\pm \mathrm{SD}, \mathrm{n}=3$ ) are summarized as follows; for DXR, $\mathrm{AUC}_{0-420} 725.6 \pm 26.1 \mu \mathrm{g} \mathrm{hr} / \mathrm{l} ; \mathrm{T}_{1 / 2}$ $8.3 \pm 0.8 \mathrm{hr} ;$ CL $6.9 \pm 2.1 \mathrm{l} / \mathrm{hr} / \mathrm{kg} ; \mathrm{V}_{\mathrm{dss}} 15.3 \pm 3.1 \mathrm{l} / \mathrm{kg}$. For DXR-ol, $\mathrm{C}_{\max } 53.9 \pm 8.1 \mu \mathrm{g} / \mathrm{l} ; \mathrm{T}_{\max }$ $23.3 \pm 5.8 \mathrm{~min} ; \mathrm{AUC}_{0-420} 201.2 \pm 26.1 \mu \mathrm{g} \mathrm{hr} / \mathrm{l} ; \mathrm{T}_{1 / 2} 9.2 \pm 1.3 \mathrm{hr}$. These results indicate the higher conversion of DXR to DXR-ol when DXR was administered as free form compared to the pegylated liposomal form [28]. The results are consistent with previous studies for the free doxorubicin formulations [11,24]. Consequently, the obtained results demonstrate that the proposed HPLC-PO-CL method is suitable for pharmacokinetic studies of DXR and its metabolite DXR-ol in rats.

\section{Conclusion}

A highly sensitive and selective HPLC-PO-CL method has been developed and validated for the simultaneous determination of DXR and DXR-ol in rat plasma. The method was based on the unique photosensitization and fluorescence characteristics of DOX and DOX-ol, which enabled the use of aryloxalate as a single post-column CL reagent that provides advantages on the stability of CL reagent and subsequently reproducibility of the method in addition to the expected decrease of background noise. Because only fluorescent compounds with quinone moiety can give a response in our method, the high selectivity is expected for the proposed method. Furthermore, sample processing procedure involves a single protein precipitation step with a sample size of $50-\mu \mathrm{L}$ which enabled many sampling points. Besides, it can detect accurately the low plasma concentrations due to its high selectivity and sensitivity which was comparable or even better than most of the published methods [6, 8-25]. These advantages make our method strongly needed in cases where it is difficult to take larger sample volume with small sample concentration. Finally, the proposed method has been successfully applied to study pharmacokinetic profiles of DXR and DXR-ol in rats after a single dose of DXR.

\section{References}

[1] G.N. Hortobagyi, , Drugs 54 (Suppl. 4) (1997) 1.

[2] D.L. Keefe, , Semin. Oncol. 28 (Suppl.12) (2001) 2. 
[3] G. Minotti, R. Ronchi, E. Salvatorelli, P. Menna, G. Cairo, Cancer Res. 61 (2001) 8422.

[4] A. Gavenda, J. Sevcik, J. Psotova, P. Bednar, P. Bartak, P. Adamovsky, V. Simanek, Electrophoresis, 22 (2001) 2782.

[5] A. Eder, J. Chen, E. Arriaga, Electrophoresis, 27 (2006) 3263.

[6] T. Pérez-Ruíz, C. Martínez-Lozano, A. Sanz, E. Bravo, Electrophoresis, 22 (2001) 134.

[7] N. J. Reinhoud, U. R. Tjaden, H. Irth, J. Vander Greef, J. Chromatogr., 574 (1992)327.

[8] R. Rahmani, P. Gil, M. Martin, A. Burand, J. Barbet, J. Cano, J. Pharm. Biomed. Anal., 1 (1983) 301.

[9] I. Badea, L. Lazar, D. Moja, D. Nicolescu, A. Tudose, J. Pharm. Biomed. Anal., 39 (2005) 305.

[10] O. T. Fahmy, M. A. Korany, H. M. Maher, J. Pharm. Biomed. Anal., 34 (2004) 1099.

[11] Q. Zhou, B. Chowbay, J. Pharm. Biomed. Anal., 30 (2002) 1063.

[12] C. M. Gilbert, R. P. McGeary, L. J. Filippich, R. Norris, B. Charles, J. Chromatogr. B, 826 (2005) 273.

[13] S. Emara, I. Morita, K. Tamura, S. Razee, T. Masujima, H. Mohamed, S. El-Gizawy, N. El-Rabbat, Talanta, 41 (1994) 1973.

[14] S. Emara, I. Morita, K. Tamura, S. Razee, T. Masujima, H. Mohamed, S. El-Gizawy, N. El-Rabbat, J. Liq. Chrom. \& Rel. Technol., 21 (1998) 681.

[15] S. N. Mahnik, B. Rizovski, M. Fuerhacker, R. M. Mader, Chemosphere, 65 (2006) 1419.

[16] L. Alvarez-Cedron, M. L. Sayalero, J. M. Lanao, J. Chromatogr. B, 721 (1999) 271.

[17] S. Fogli, R. Danesi, F. Innocenti, A. Di Paolo, G. Bocci, C. Barbara, M. Del Tacca, Ther. Drug Monit., 21 (1999) 367.

[18] N. laubrock, G. Hempel, P. Schulze-Westhoff, G. Wurthwein, S. Flege, J. Boos, Chromatographia, 52 (2000) 9.

[19] P. de Bruijn, J. Verweij, W. J. Loos, H. J. Kolker, A. S. T. Planting, K. Nooter, G. Stoter and A. Sparreboom, Anal. Biochem., 266 (1999) 216.

[20] J. van Asperen, O. van Tellingen, J. H. Beijnen, J. Chromatogr. B, 712 (1998) 129.

[21] L. H. Reddy, N. Meda, R. R. Murthy, Acta Pharm. 55 (2005) 81.

[22] K. Imai, A. Nishitani, Y. Tsukamoto, Chromatographia, 24 (1987) 77.

[23] R. Ricciarello, S. Pichini, R. Pacifici, I. Altieri, M. Pellegrim, A. Fattorossi, P. Zuccaro, J. Chromatogr. B, 707 (1998) 219.

[24] R. DiFrancesco, J. J. Griggs, J. Donnelly, R. DiCenzo, J. Chromatogr. B, 852 (2007) 
545.

[25] F. Lachatre, P. Marquet, S. Ragot, J. M. Gaulier, P. Cardot, J. L. Dupuy, J. Chromatogr. B, 738 (2000) 281.

[26] C. Sottani, G. Tranfo, M. Brettinelli, P. Faranda, M. Spagnoli, C. Minoia, Rapid Commun. Mass Spectrom., 18 (2004)2426.

[27] Y. Yang, Talanta, 71 (2007)596-604.

[28] Y. Liu, Y. Yang, X. Liu, T. Jiang, Talanta, 74 (2008) 887-895.

[29] R. D. Arnold, J. E. Slack, R. M. Straubinger, J. Chromatogr. B, 808 (2004) 141.

[30] A. M. Garcia-Campana, W. R. Baeyens, Chemiluminescence in Analytical chemistry, M. Dekker, New York, 2001, Chapter 7, p.141.

[31] S. Ahmed, S. Fujii, N. Kishikawa, Y. Ohba, K. Nakashima, N. Kuroda, J. Chromatogr. A, 1133 (2006) 76.

[32] S. Ahmed, N. Kishikawa, K. Nakashima, N. Kuroda, Anal. Chim. Acta, 591 (2007) 148.

[33] J. R. Poulsen, J. W. Birks, Anal. Chem., 62 (1990) 1242-1251.

[34] T. Pérez-Ruíz, C. Martínez-Lozano, V. Tomás,J. Martín, Analyst, 124 (1999) 197.

[35] Department of Health and Human Services, Food and Drug Administration, Guidance for Industry on Bioanalytical Method Validation, Fed. Regist. 66 (2001) 28526.

[36] K. Gollnick and S. Held, J. Photochem. Photobiol. A: Chem., 70 (1993)135.

[37] L. Gamiz-Garcia, A. M. Garcia-Campana, F. Ales Barrero, L. Cuadros Rodriguez, Anal. Bioanal. Chem., 377 (2003)281.

[38] T. Pérez-Ruíz, C. Martínez-Lozano, M. D. Garcia, J. Martín, J. Chromatogr. A, 1141 (2007) 67.

[39] P. Prados, T. Santa, H. Homma, and K. Imai, Anal. Sci., 11 (1995) 575.

[40] K. Imai, H. Nawa, M. Tanaka, H. Ogata, Analyst, 111 (1986) 209.

\section{Figure caption}

Fig. 1. Chemical structures of doxorubicin and its metabolite doxorubicinol.

Fig. 2. HPLC -PO-CL system for the determination of DXR and DXR-ol.

P1, pump 1; P2, pump 2; I, injector; Column, Cosmosil 5C18-AR-II (150 x 2 mm, i.d.); Cooling unit, oven equipped with cold insulator; L, low-pressure mercury lamp; RC, reaction coil (2.0 m x 0.25 mm, i.d.); $\mathrm{M}$, mixing tee; $\mathrm{NC}$, noise cleaner; $\mathrm{D}$, CL detector; Rec, 
recorder.

Fig. 3. Effects of hydrogen atom-donating substrates on (A) CL intensity and (B) S/N ratio. Samples were 500 nM DXR and DXR-ol.

Fig. 4. Effects of $\mathrm{pH}$ of imidazole-TFA buffer on (A) CL intensity and (B) S/N ratio. Samples were 500 nM DXR and DXR-ol.

Fig. 5. Chromatogram of (A) a mixture of $500 \mathrm{nM}$ standard DXR and DXR-ol (B) rat plasma collected after $60 \mathrm{~min}$ of single dose administration of $5 \mathrm{mg} / \mathrm{kg}$ DXR and analyzed by the proposed HPLC-PO-CL method.

Fig. 6. Concentration-time profiles of DXR and its metabolite DXR-ol in rat plasma after a single dose administration of $5 \mathrm{mg} / \mathrm{kg}$ DXR; Data expressed as mean $\pm \mathrm{SD}(\mathrm{n}=3)$. 
Table 1

Retention times, calibration curves and detection limits of DXR and DXR-ol

\begin{tabular}{|c|c|c|c|c|c|c|}
\hline \multirow[b]{2}{*}{ Sample } & \multirow{2}{*}{$\begin{array}{c}t_{\mathrm{R}} \\
(\mathrm{min})\end{array}$} & \multicolumn{4}{|c|}{ Calibration curve $^{\mathrm{a}}$} & \multirow{2}{*}{$\begin{array}{c}\text { Detection limit }^{\mathrm{b}} \\
\text { fmol/injection } \\
\text { (nM) }\end{array}$} \\
\hline & & $\begin{array}{c}\text { Range } \\
(\mathrm{nM})\end{array}$ & $\begin{array}{l}\text { Slope }^{\mathrm{c}} \\
( \pm \mathrm{SE})\end{array}$ & $\begin{array}{c}\text { Intercept }^{\mathbf{c}} \\
( \pm \mathrm{SE})\end{array}$ & $\overline{\mathbf{r}}$ & \\
\hline DXR & 14.1 & $2-1000$ & $\begin{array}{c}0.09 \\
( \pm 0.01)\end{array}$ & $\begin{array}{c}6.5 \\
( \pm 0.52)\end{array}$ & 0.998 & $4.5(0.90)$ \\
\hline DXR-ol & 9.2 & $2-1000$ & $\begin{array}{c}0.09 \\
( \pm 0.02)\end{array}$ & $\begin{array}{c}7.7 \\
( \pm 0.65)\end{array}$ & 0.999 & $3.8(0.76)$ \\
\hline
\end{tabular}

${ }^{\text {a }} \mathrm{CL}$ intensity(arbitrary unit) versus concentration (nM).

${ }^{\mathrm{b}}$ Detection limit at a $\mathrm{S} / \mathrm{N}$ ratio of 3

${ }^{c}$ Data presented as mean \pm SE of three experiments

Table 2

Accuracy and precision of the proposed method in rat plasma

\begin{tabular}{cccccc}
\hline Sample & \multirow{2}{*}{$\begin{array}{c}\text { Concentration } \\
(\mathrm{nM})\end{array}$} & \multicolumn{2}{c}{ Intra-day assay $(\mathrm{n}=5)$} & \multicolumn{2}{c}{ Inter-day assay $(\mathrm{n}=3)$} \\
\cline { 3 - 6 } & \multirow{2}{*}{ Accuracy $(\%)$} & $\begin{array}{c}\text { Precision } \\
(\text { RSD\% })\end{array}$ & $\begin{array}{c}\text { Accuracy } \\
(\%)\end{array}$ & $\begin{array}{c}\text { Precision } \\
(\text { RSD\% })\end{array}$ \\
\hline DOX & 50 & 95.2 & 7.6 & 92.3 & 8.9 \\
& 250 & 93.3 & 5.7 & 101.5 & 7.5 \\
& 1000 & 100.7 & 4.6 & 97.4 & 3.5 \\
DOX-ol & 50 & 90.4 & 5.9 & 100.1 & 6.4 \\
& 250 & 102.5 & 2.5 & 94.1 & 5.7 \\
& 1000 & 96.1 & 3.9 & 91.2 & 4.8 \\
\hline
\end{tabular}


Table 3

Stability of DXR and DXR-ol in rat plasma

\begin{tabular}{ccccc}
\hline & \multicolumn{3}{c}{ Percentage of initial concentration $(\%)(\mathrm{n}=3)$} \\
\cline { 2 - 5 } Condition & \multicolumn{2}{c}{ DXR } & DXR-ol \\
& High $(1000 \mathrm{nM})$ & Low $(50 \mathrm{nM})$ & $\begin{array}{c}\text { High }(1000 \\
\text { nM) }\end{array}$ & Low $(50 \mathrm{nM})$ \\
\hline Three times freeze-thaw & 91.9 & 89.6 & 90.0 & 87.4 \\
Room temperature $(1 \mathrm{hr})$ & 102.3 & 96.8 & 98.9 & 97.2 \\
Room temperature $(5 \mathrm{hr})$ & 96.4 & 98.8 & 93.1 & 90.5 \\
Room temperature $(24 \mathrm{hr})$ & 85.0 & 82.7 & 81.9 & 80.3 \\
Refrigeration over night $\left(4{ }^{\circ} \mathrm{C}\right)$ & 93.0 & 91.5 & 90.6 & 87.4 \\
Freezer at $-30{ }^{\circ} \mathrm{Cfor}$ three weeks & 90.2 & 87.6 & 88.4 & 86.1 \\
\hline
\end{tabular}


Fig. 1<smiles>COc1cccc2c1C(=O)c1c(O)c3c(c(O)c1C2=O)C[C@@](O)(C(=O)CO)C[C@@H]3OC1CC(N)C(O)C(C)O1</smiles>

Doxorubicin (DXR)<smiles>COc1cccc2c1C(=O)c1c(O)c3c(c(O)c1C2=O)C[C@@](O)(C(O)CO)C[C@@H]3OC1CC(N)C(O)C(C)O1</smiles>

Doxorubicinol (DXR-ol)

Fig. 2

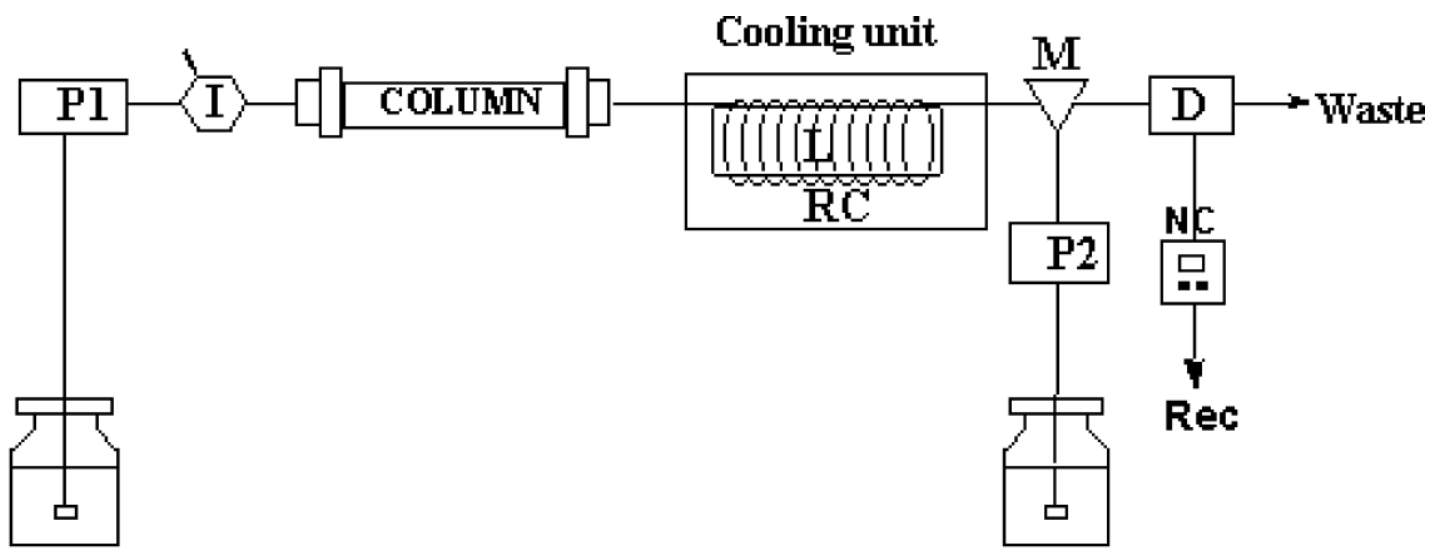

Mobilephase : $50 \mathrm{mM}$ imidazole $T F A$ buffer $\mathbf{p H} 6.8$ )/acetonitrile/ethanol CL reagent : $05 \mathrm{mM}$ TDPO in $(=55: 35: 10, \mathrm{v} / \mathrm{v} / \mathrm{v})$ contain $20 \mathrm{mM}$ SDS acetonitrile (llow rate $=0.7 \mathrm{ml} / \mathrm{min}$ ) (flow rate $=03 \mathrm{ml} / \mathrm{min}$ ) 
Fig. 3
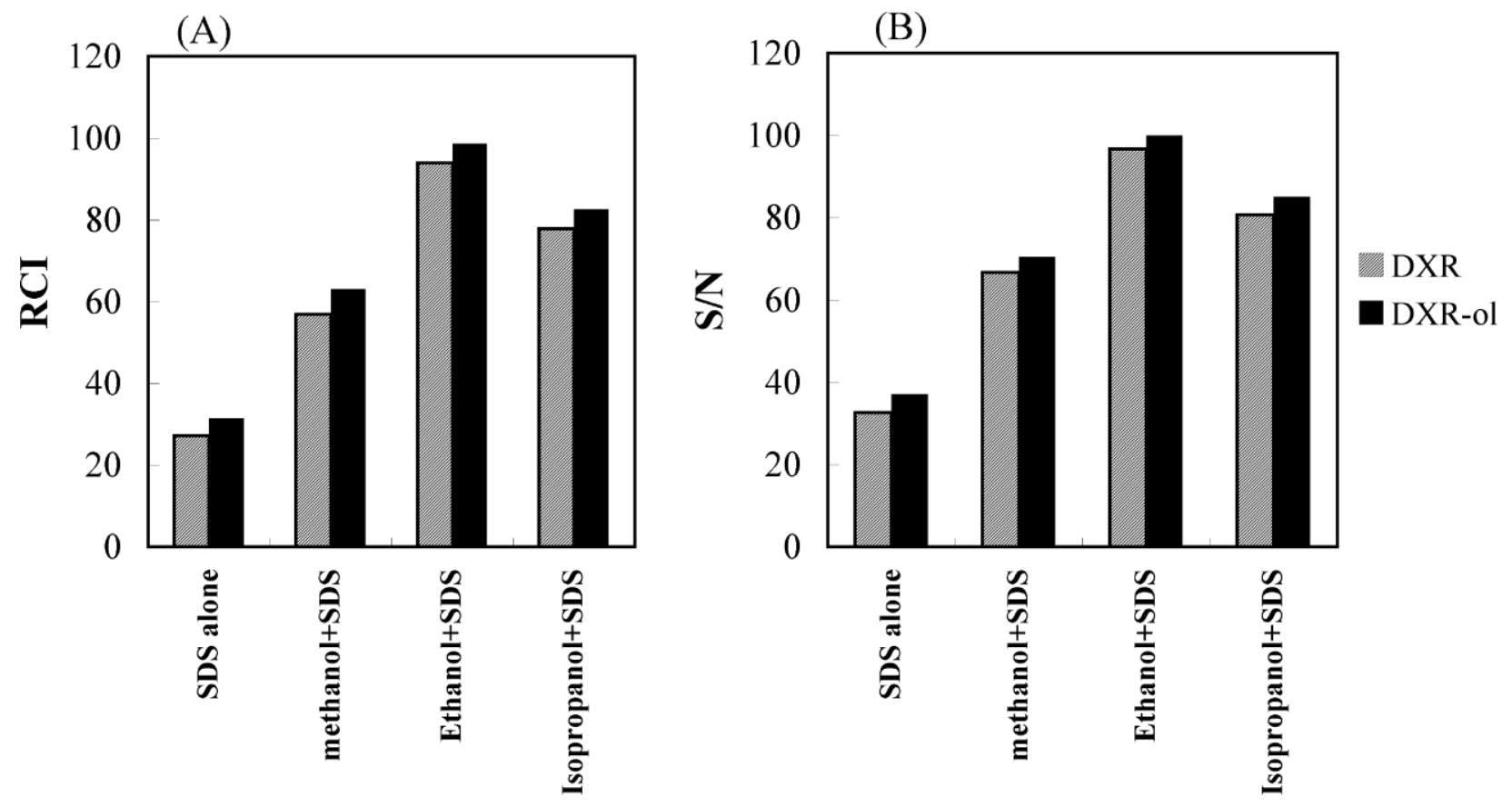

Fig. 4
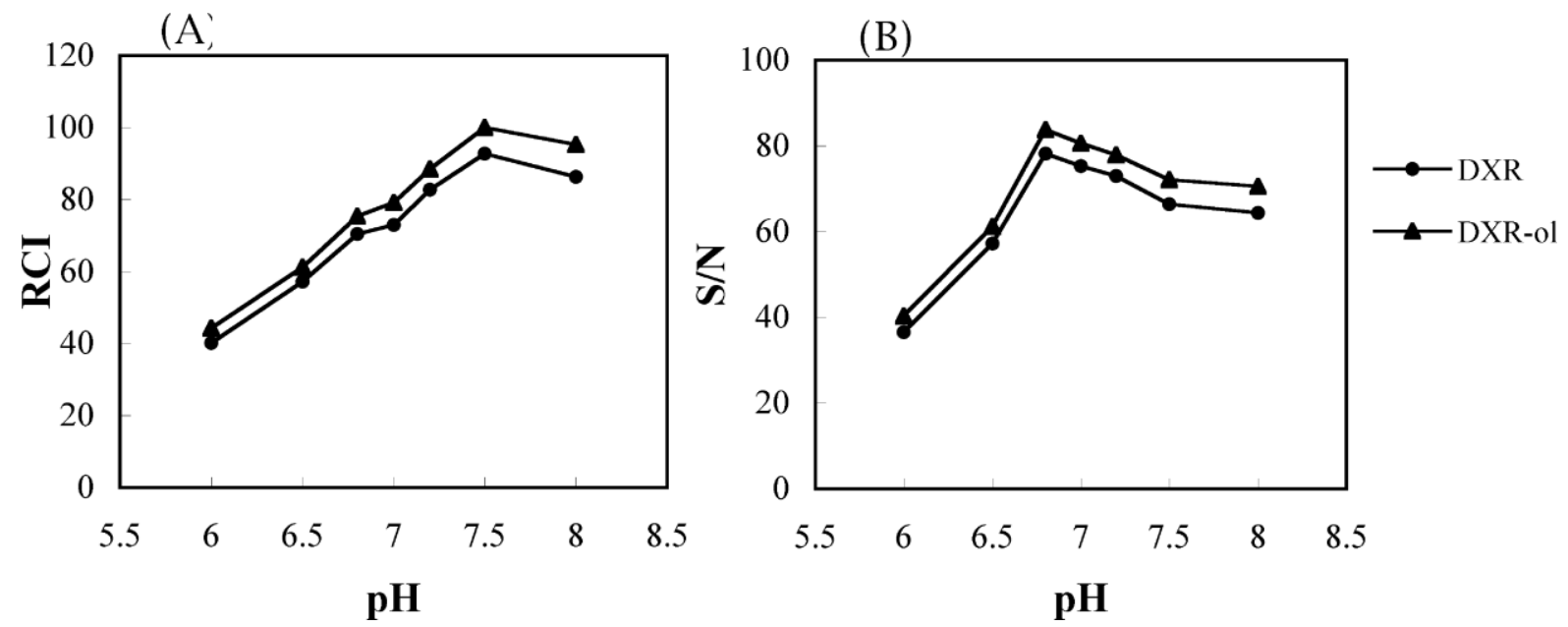
Fig. 5
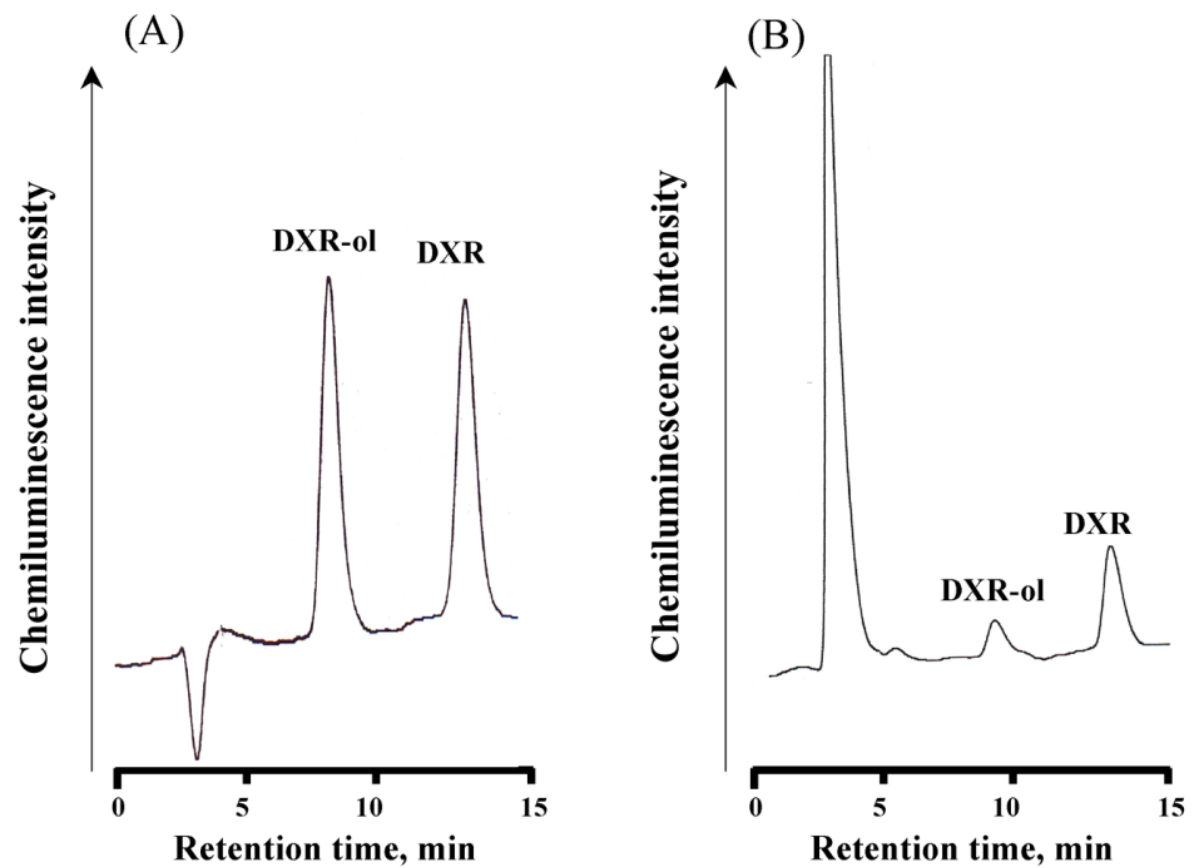

Fig. 6

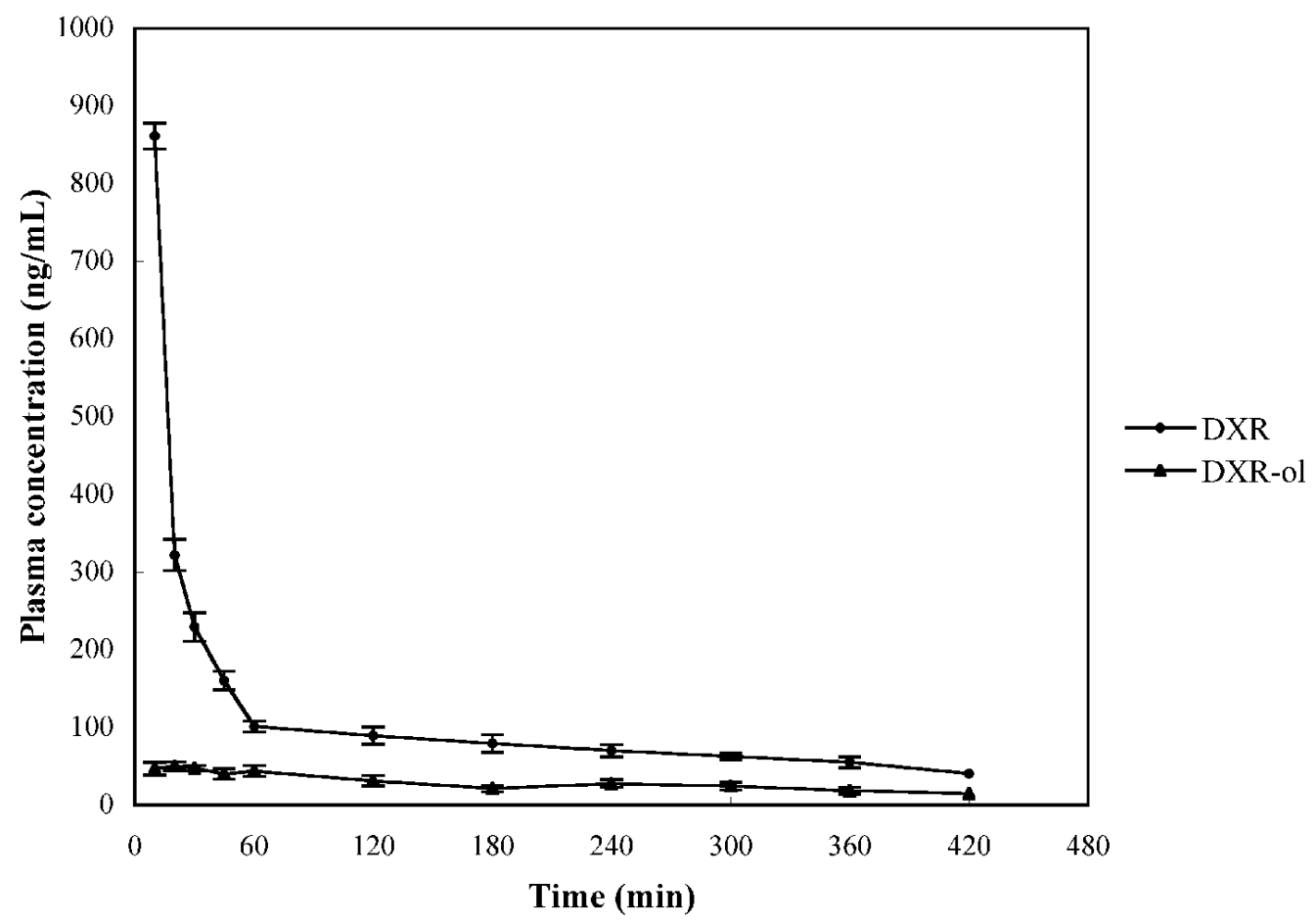

